\title{
ANALYSIS OF THE D-ZERO CRANE RAIL AS A SUPPORT FOR A HORIZONTAL LIFELINE
}

D-ZERO ENGINEERING NOTE \# 3823.000 EN-519

Author: Herman Cease, PPD/ETT/D-Zero Mech.

March 2, 2000

Approved By:

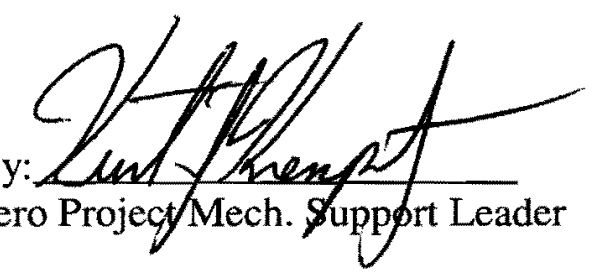




\section{Summary:}

The D-Zero crane rail is analyzed for use as an anchor support for a one person Horizon ${ }^{\mathrm{TM}}$ Horizontal Lifeline system that will span the pit area at D-Zero assembly hall. The lifeline will span $75 \mathrm{ft}$ across the pit area, will be located out of the travel of the crane and above the concrete lentil wall.

\section{Analysis:}

The crane rail dimensions are 29.5 inches vertical height, 10.625 inch flange width and 0.875 inch flange thickness on the lower flange. The unsupported span is 25 feet. The maximum tension on the cable for the $75 \mathrm{ft}$ span with a one person support system is 3,500 lbf total - data from MSA-ROSE mfg. The maximum lateral force at the Lifeline anchor positions on the crane rail then is $1,750 \mathrm{lbf}$. Assuming only the flange section contributes to strength and the anchor is attached at the midpoint of the rail, the expected stresses and deflections on the rail are:

$$
\begin{aligned}
& S=-W l / 4 Z \\
& D=W l^{3} / 48 E I
\end{aligned}
$$

Where,

$$
\begin{aligned}
& \mathrm{W}=1,750 \mathrm{lb} \\
& \mathrm{L}=12.5 \text { feet }=150 \text { inches } \\
& \mathrm{E}=\text { modulus of steel }=28 \mathrm{e} 6 \mathrm{psi} \\
& \mathrm{Z}=\mathrm{bh}^{2} / 3=0.875^{*} 10.625^{2} / 3=32.9 \mathrm{in}^{3} \\
& \mathrm{I}=\mathrm{bh}^{3} / 12=0.875^{*} 10.625^{3} / 12=87 \mathrm{in}^{4}
\end{aligned}
$$

Max stress at the loading point $=1,995$ psi

Max deflection at the loading point $=0.047$ inches

The maximum allowable stress is $1 / 3$ yield strength of the rail or $1 / 3 * 33,000$ psi $=$ $11,000 \mathrm{psi}$. This would allow a maximum of five - one person lifeline support systems.

The minimum breaking strength of the $3 / 8$ " $7 \times 19$ galvanized wire rope is $14,400 \mathrm{lbf}$. The lateral load at the anchor position is then $7,200 \mathrm{lbf}$. Stress in the mounting rail, assuming only the flange section contributes is 8,200 psi indicating the rating for the steel rope is exceeded before exceeding the maximum allowable stresses in the crane rail.

Consideration of Crane loads:

The lifeline system puts a horizontal load on the crane rail. This load is perpendicular to any loads on the crane rail due to at the hook loads. Therefore, at the hook loads and loads occurring during a falling arrest are not additive on the crane support rails.

\section{Conclusions:}

The crane rail is a suitable anchor for a one person Horizon ${ }^{\mathrm{TM}}$ Horizontal Lifeline system. The expected stress on the rail is $1,995 \mathrm{psi}$ which has a factor of safety of 5.5 on the allowable stress. The anchor position is located 18" away from the concrete lentil wall and out of the travel of the overhead crane. 


\section{HORIZONTM \\ Horizontal Lifeline Anchorage Connector Subsystem}

\section{BELOW BEAM MULTPLE SPAN SUBSYSTEM}

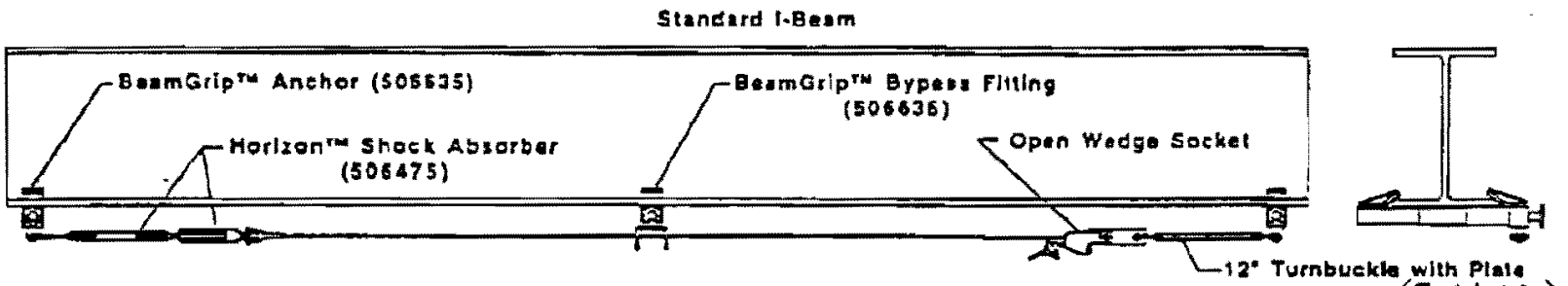

The Horizon ${ }^{\mathrm{TM}}$ Below Beam Multiple Span Horizonial Lifeline Specifications $(50<262)$

\begin{tabular}{|c|c|}
\hline $\begin{array}{l}\text { Litoline Length } \\
\text { Unsupporied Span } \\
\text { Cable } \\
\text { Compatibility } \\
\text { Suppon Equipment } \\
\text { Min Anchorage Strength }\end{array}$ & $\begin{array}{l}20 \text { is } 280 \text { feet in spans up to } 40 \text { teet } \\
40 \text { teet maximun } \\
318^{*} 7 \times 19 \text { Galvanized wire rope - minimum breaking strength fated al } 14.400 \mathrm{lb} \\
\text { Fits most standard l-beams } \\
\text { Requires the use of an energy absorbing lanyard that limits fall arrest forces to } 900 \mathrm{lbl} \\
12.500 \text { lbf lateral strength required for installation }\end{array}$ \\
\hline
\end{tabular}

PRODUCT DESCRIPTION: The Horizon'M Horizontal Lifeline, with integral in line shock absorber, provides complete freedom of movement for the entire length of the lifeline without having 10 detach the workers' personal fall arrest system. The Horizon ${ }^{\top M}$ Shock Absober reduces line tension by up to $38 \%$ during a single fall condition (see Anchorage Loading Chart below).

Hortzon ${ }^{\text {rm }}$ Horlzontal Lifellne Anchorage Loading Chart (140 ft Length - $20 \mathrm{ft}$ Span)

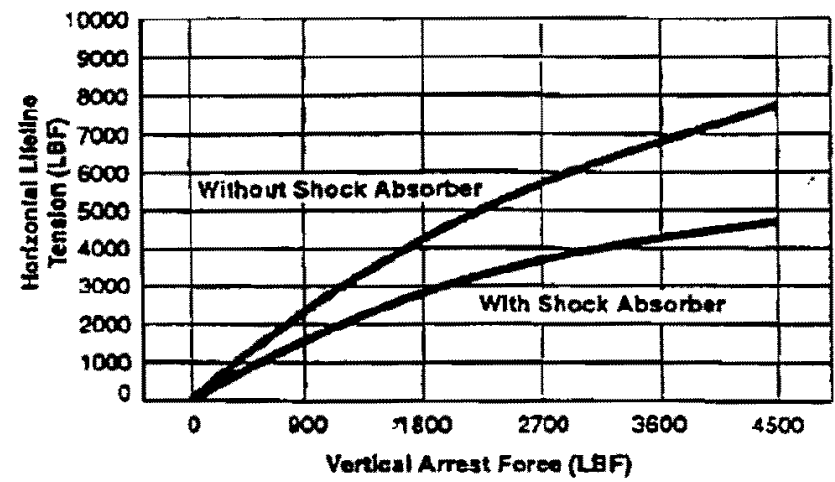

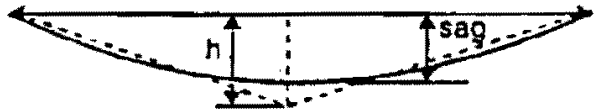

Horizon ${ }^{\text {rm }}$ Shock Absorber Load Chart

(140 Ht Length - $20 \mathrm{H}$ Span) Tabla 1

\begin{tabular}{|c|c|c|c|}
\hline $\begin{array}{l}\text { Input } \\
\text { Foree } \\
\text { (F1) }\end{array}$ & $\begin{array}{c}\text { Anchorage } \\
\text { Load } \\
\text { (F2) }\end{array}$ & $\begin{array}{l}\text { Anchorage } \\
\text { Losd } \\
\text { (F3) }\end{array}$ & $\begin{array}{c}\text { Change at } \\
\text { Elevation } \\
\text { (h) }\end{array}$ \\
\hline $\begin{array}{ll}1 & \text { Person } \\
2 & \text { Person } \\
3 & \text { Person } \\
4 & \text { Person } \\
5 & \text { Person }\end{array}$ & $\begin{array}{l}1,860 \mathrm{lbt} \\
3,000 \mathrm{lbt} \\
3,460 \mathrm{lbt} \\
4,000 \mathrm{lbt} \\
4,720 \mathrm{lbf}\end{array}$ & $\begin{array}{l}2,280 \mathrm{lbl} \\
3,840 \mathrm{lbf} \\
4,480 \mathrm{lbl} \\
6,880 \mathrm{lbl} \\
7,640 \mathrm{lbl}\end{array}$ & $\begin{array}{l}37.75 \text { inches } \\
38.00 \text { inches } \\
41.50 \text { inches } \\
42.00 \text { inches } \\
48.00 \text { inches }\end{array}$ \\
\hline
\end{tabular}

Based on $140 \mathrm{~h}$ langeth with supported spans of $20 \mathrm{n}$ F1 - Lanyard Energy Absorber rated al $800 \mathrm{Bl}$ F2 - With Horizon'" Shock Absorbor F3 - Withour Horizon't Shock Absorbar

Rose Manufacturing Company - 2250 South Tejon Street - Englewood, Co 80110-1000 - USA Phone 303-922-6246 - Toll free phone 1-800-722-1231 - Fax 303-934-9960 


\section{HORIZONTM}

\section{Horizontal Lifeline Subsystem}

\section{COWPONENT SPECIFICATIONS- HORIZONTAL LIFELINE SUBSYSTEM}

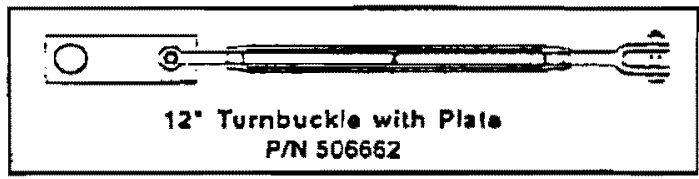

12" Turnbuckle with Plate - Part Number 506662

Material High lensile alloy steel

Thread Course. $1 / 2$ inch

Pange Up to 12 inch adiusiment

Use Line tensioning

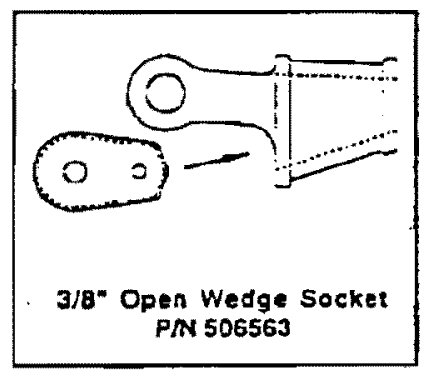

$3 / 8^{\circ}$ Open Wedge Socket -Pan Number 505563

Use Secures one end of HLL

Sirength $\quad 12.500 \mathrm{lbl}$

Features Adjustable for line length positioning

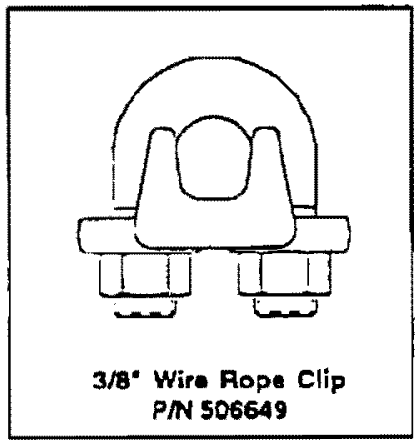

3/8" Wire Rope Clip - Pant Number 506649

Use

Socures wire ropa end

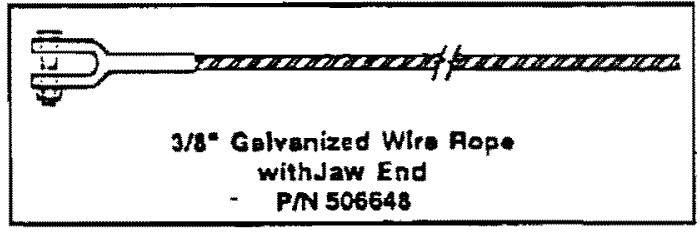

3/8" Galvanized Wire Rope with Jaw end- Part Number 605548

Type

Strength

Length
$7 \times 19$ Galvanized steel cable

14,400 tot tension

20 feet minimum depanding on application

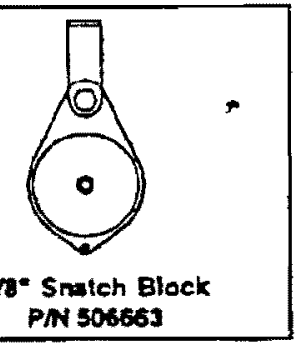

3/8" Snatch Block Pulley - Part Number 506663

Use Connects Rose Dyna-Locks 8 Dynaevacs to 3/8" dia harizontal Ifeline. Can be use only on single span systems. 


\section{HORIZONTM}

\section{Horizontal Lifeline Subsystem}

\section{COMPONENT SPECIFCATIONS - HORIZONTAL LIFELINE SUBSYSTEM}

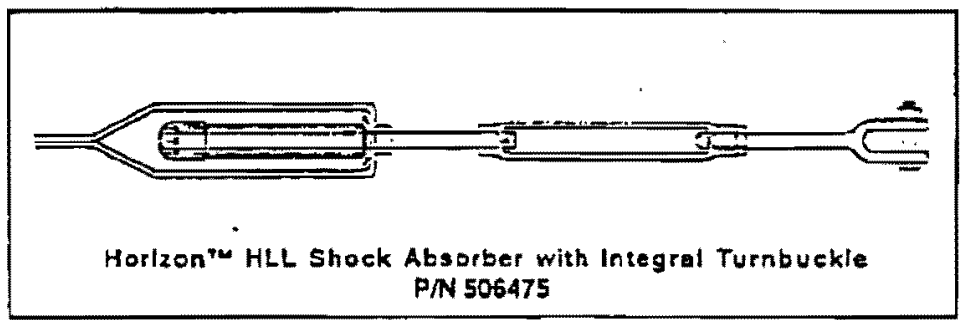

The Horizon'm Shock Absorber assembly otters easy tensioning adjustment and constent load energy absorption to provide lower anchorage forces.

$\begin{array}{ll}\text { Material } & \text { High tensile alloy \& stainless } \\ & \text { steel } \\ \text { Sirength } & 12,500 \text { bt minimum } \\ \text { AJjustment } & 6 \text { inches nominal } \\ \text { Lengh } & 24 \text { inches nominal }\end{array}$
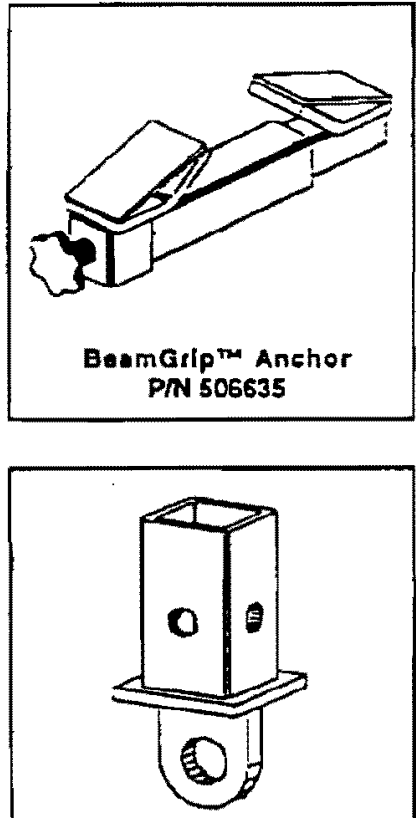

BeamGilp End Fitting P/N 606637

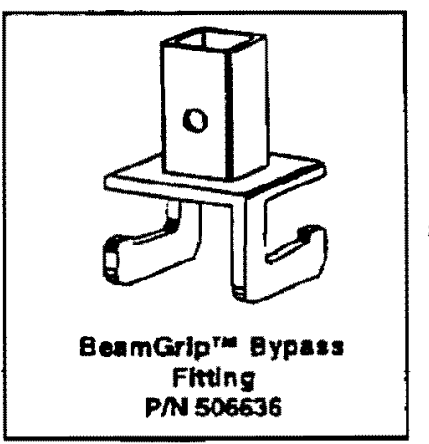

The BeamGrip ${ }^{2 m}$ Anchor essembly allows quick and easy installation to standard beam sizes while offering over 12,500 bf of latera! ancherage strength.

Material

Strength

I Beam Compatibility

System Compatibility

Cabon sleel, yellow zinc plated

$12.500 \mathrm{bl}$

Wide range of sizes to accommodale most beams

SeamGrip ${ }^{\top m}$ End Fing. Pan Number 506637

BeamGrip ${ }^{T M}$ Eypass Fiting, Part Number 506636

The BeamGrip ${ }^{\text {m }}$ End Fitting is used in corjunction with the BeamGrip Anchor, $^{\text {m }}$ pictured above, and is designed to allow orientation for ancho: age loading in 3 directions.
Material
Strength
Carbon sleet, yellow zinc plated
Compationity
BeamGriptM Anchor assambly, Part Number 506635

Nole: (Must use linkage components approved for use with the Horizon ${ }^{\mathrm{MM}}$ Horizontal Lifeline oniy.)

The BeamGriptm Bypass Fitting allows a worker to bypass an intermediate span support without disconnecting from the horizontal ifeline.
Material
Strength
Carbon steel, yellow zinc plated
5,000 lbi vertical
3,600 bo lateral
Compatioity
Beamarip Th Anctor assembly, Par Number 506635 
HORIZON'M

\section{Horizontal Lifeline Subsystem}

\section{COMPONENT SPECIFICATIONS - PERSONNEL FALL ARAEST SUBSYSTEM}

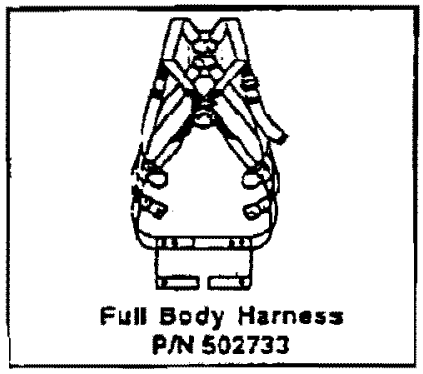

Full Body Harness - Pant Number 502733

Sizes

System Usage

D*ring locations

Weight

Standards

Capacity

Material

$x$-small, slandard, $x$-large, super $x$-large

Fall arrest. controled descent. ladder climbing, confined

space entry and exit and worker positioning

Back, chest and 2 hip

$3.5 \mathrm{lbs}$

Meets ANSI Z359.1, ANSI A 10.14, CSA Z259.10, OSHA

510 los including clothes, tools and other user bome objects

Nylon (polester 502734)
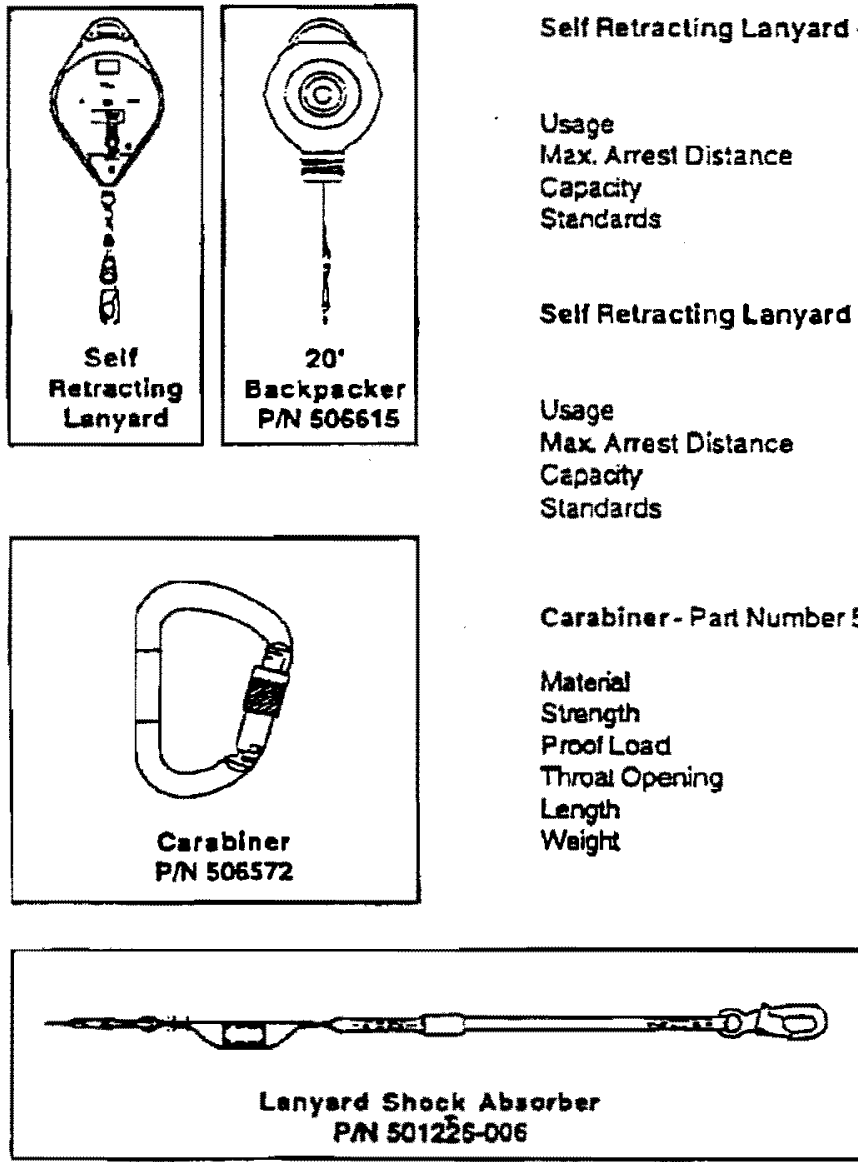

Material

Strength

Prool Load

Throal Opering

Length

Waight

Max Arrest Distance

Capacity

Standards

\section{4 inches}

310 lbs including clothes, 100 s and other user bome objects Meets ANSI ZJ59.1, ANSI A 10.14, OSHA

$506208 \cdot 30$ Meter Dyna-Lock $506204 \cdot 16$ Meler Oyna-Lock

Single span systems

24 inches

310 los including clothes, tools and other user buinte ujecis Meets ANSI 2359.1. ANSI A 10.14. OSHA

Carabiner - Pant Number 506572

\section{$5,000 \mathrm{bl}$}

Hightensile alloy steel

3.600 bof

.675 inch minimum

3.7 nominal

$0.6 \mathrm{cos}$

Lanyard Shock Absorber - Part Number -

501226-006

$\begin{array}{ll}\text { Max Arest Distance } & 42 \text { inches } \\ \text { Max Arrest Force } & 900 \text { Dot } \\ \text { Cepacity } & 130 \text { to } 310 \text { los inchuding } \\ & \text { clothes, lools and other user } \\ & \text { bome objects } \\ \text { Material } & \text { Nylon or polyester } \\ \text { Compationty } & \text { Equipped with snaphook or } \\ & \text { wobbed loop } \\ \text { Slandards } & \text { Meets ANSIZZ359.1. ANSI } \\ & \text { A10.14, OSHA }\end{array}$

Max Arrest Distance 42 Inches

Max Arrest Force $\quad 900 \mathrm{Bt}$

I30 to 310 los incuuding

bome objects

Equipped with snaphook or

Meets ANSI Z359.1, ANSI

A10.14, OSHA 


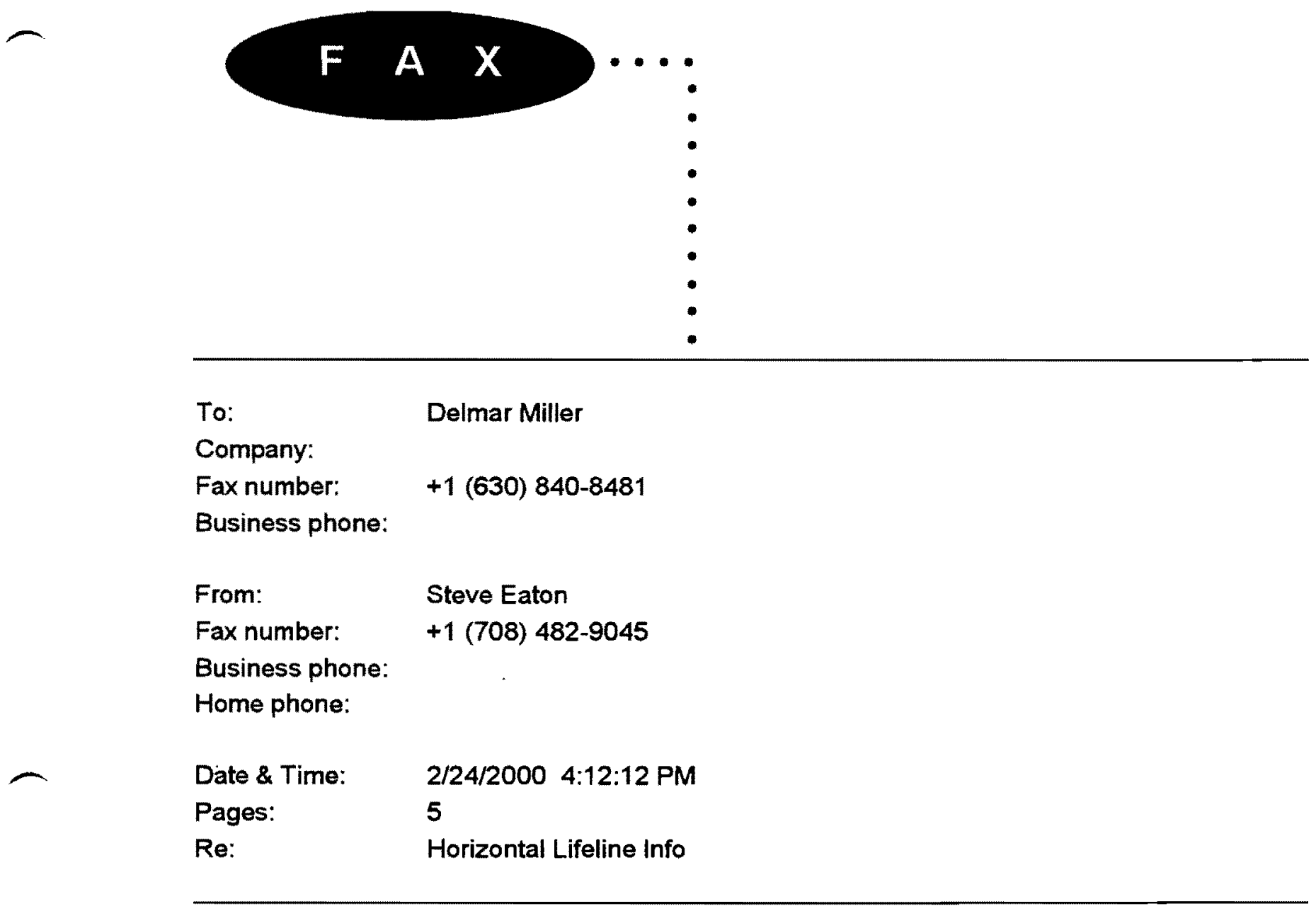


Rose Manufacturing C 2250 S. Tejon st. Englewaod, $C O$

80110-1000

\section{TELEFAX TRANSMISSION}

Phone [303] 922-624t Fax [303] 934-996.

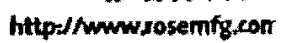

To: Delmar or Hemman e Ferme From: Mart Back

Fax: $6308408481 \quad$ Pages: z

Phone:

Date:

Re: HLL Spes. CC:

$\square$ Uigent $\square$ For Reviow $\square$ Please Comment $\square$ Please Reply $\square$ Please Rocyclo


Fermi Lab

Delmar Miller / Herman Sites

630-840-8481 fax
From Mark Blake

Phone 303-975-2315

Fax 303-934-9960

E-Mail Mark.Blake@MSAnet.com

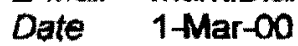

Re Calculations for a 75 foot Horizontal Lifoline
Rose Manufacturing $\mathrm{Co}$. 2250 S. Tejon St Englewood, $c 0$ 8ono-1000

Phone [303] 922-6246 Fax [303] $934-9960$ https//unwwrosemfg-com

Dear Sirs,

in response to a request made by Steve Eaton of Elliott sales here are the specifications for the horizontal lifeline system outlined below.

System Length -75 feet

No. on Spans - 1

No. of Workers -1

Force per Person - 900 lbs.

MSA Rose HLL Shock Absorber - Yes

\section{Specification for unloaded system}

Initial line tension $\mathbf{- 5 1 4}$ lbs.

Sag Distance for horizontal $-4^{n}$

\section{Specification for loaded system}

Workers arrested -1

Maximum Cable Tension $-3,500$ lbs.

Maximum Cable Drop - 5.0 feet

Total Fall Distance based on use of self-retracting lanyard and Full Body Hamess- 8.0 feet

Please feel free to call me with any additional questions that you may have.

Regards,

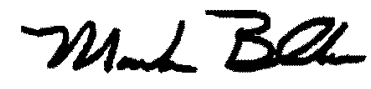

Mark Blake 


\section{ADDENDUM TO:}

\section{ANALYSIS OF THE D-ZERO CRANE RAIL AS A SUPPORT FOR A HORIZONTAL LIFELINE}

D-ZERO ENGINEERING NOTE \# 3823.000 EN-519

Author: Herman Cease, PPD/ETT/D-Zero Mech.

March 3, 2000

Approved By:

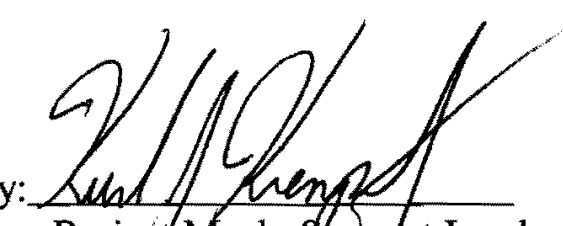

PPD/ETT/D-Zero Projecf Mech. \$upport Leader 


\section{Addendum:}

The crane rail is a suitable anchor for a one person Horizon ${ }^{\mathrm{TM}}$ Horizontal Lifeline system. The expected stress on the rail is 1,995 psi which has a factor of safety of 5.5 on the allowable stress. If a multi-person system is required, 4 additional one person Horizon ${ }^{\mathrm{TM}}$ Horizontal Lifeline systems can be attached to the crane rail without exceeding the maximum allowable stress. Each additional system should be attached to the crane rail in a location out of the travel of the crane. 